\title{
Effect of changing NAVA levels in preterm infants with RDS
}

\author{
Julie Lefevere ${ }^{1}$, Brenda Van Delft ${ }^{1}$, Michel Vervoort ${ }^{1}$, Wilfried Cools $^{2}$, and Filip Cools $^{3}$ \\ ${ }^{1} \mathrm{UZ}$ Brussel \\ ${ }^{2}$ Vrije Universiteit Brussel \\ ${ }^{3}$ Universitair Ziekenhuis Brussel
}

December 17, 2020

\begin{abstract}
Objective: to examine the effect of changing levels of support (NAVA-level) during neurally adjusted ventilatory assist (NAVA) in preterm infant with respiratory distress syndrome (RDS) on electrical diaphragm activity. Methods: we included preterm infants admitted to the NICU, who were clinically stable and supported with NAVA, either via an endotracheal tube or via a nasal interface. Patients were recruited in the first 24 hours after the start of NAVA. Following a predefined titration protocol, NAVA levels were progressively increased starting from a level of $0,5 \mathrm{cmH} 2 \mathrm{O} / \mu \mathrm{V}$ and with increments of $0,5 \mathrm{cmH} 2 \mathrm{O} / \mu \mathrm{V}$ every 3 minutes, up to a maximum level of $4,0 \mathrm{cmH} 2 \mathrm{O} / \mu \mathrm{V}$. Respiratory and ventilation parameters were continuously recorded. Results: Ten patients were studied on NIV-NAVA and three on invasive NAVA. The NIV-NAVA patients had an average gestational age at birth of $31,4+3,2$ weeks and an average birth weight of $1615+609 \mathrm{~g}$. For all patients a breakpoint could be identified during the titration study. The breakpoint was on average at a level of $2,35+0,63 \mathrm{cmH} 2 \mathrm{O} / \mu \mathrm{V}$ in the NIV-NAVA group. With increasing NAVA levels, the respiratory rate decreased significantly. For the other parameters no clear trend was observed. No severe complications occurred. Conclusion: Preterm neonates with RDS supported with NAVA display a typical biphasic response to changing NAVA-levels with an identifiable breakpoint. This breakpoint was at a higher NAVA-level than commonly used in this clinical situation, suggesting that higher levels might be needed to optimally support preterm neonates with RDS.
\end{abstract}

\section{Introduction}

Neurally adjusted ventilatory assist (NAVA) is a form of diaphragm-triggered ventilation that uses the electrical signal from the diaphragm to proportionally assist the patient's respiration. The electrical activity of the diaphragm (Edi) is measured by electrodes in a nasogastric catheter and transmitted to the ventilator. Edi peak levels correlate with neural respiratory drive and Edi trough levels with the tonic activity of the diaphragm that prevents de-recruitment. The NAVA level is a factor that converts the Edi signal in a proportional pressure. The instantaneous change in Edi is multiplied by the NAVA level to determine the pressure above the positive end-expiratory pressure (PEEP) delivered by the ventilator. When there is no Edi-signal for a predetermined time (= apnea time), a back-up ventilation is provided. NAVA can be used as an invasive (via an endotracheal tube) or a non-invasive (via a nasal mask or nasal prongs: NIV-NAVA) ventilation mode ${ }^{1}$.

Several clinical studies have shown that NAVA and NIV-NAVA are feasible, safe and effective in preterm neonates ${ }^{2-5}$. With NAVA we can achieve optimal synchronization with the patient's own respiratory effort, which is especially challenging in preterm infants due to the rapid respiratory rate and often big air leaks ${ }^{6}$. Synchronized mechanical ventilation has several advantages in preterm infants in terms of duration of mechanical ventilation and reduced risk of air leaks ${ }^{7}$. The non-invasive use of NAVA is gaining importance as we want to avoid invasive mechanical ventilation in preterm neonates as much as possible to prevent ventilator induced lung injury, and hence, chronic lung disease of prematurity. A Cochrane review of 2016 concluded that non-invasive intermittent positive pressure ventilation (NIPPV) is superior to nasal contin- 
uous positive airway pressure (CPAP) as primary respiratory support in preterm infants with respiratory distress syndrome (RDS) to prevent intubation and invasive ventilation ${ }^{8}$.

Despite the more widespread use of NAVA, there is no evidence-based consensus on what the initial setting for the NAVA-level in preterm infants should be. As the NAVA level increases, the workload is shifted from the patient to the ventilator. This allows the work of breathing to be unloaded from the patient to the ventilator. Both in animal (rabbits) as in human studies with different populations (critically ill adults, children and preterm neonates) a 'breakpoint' (BrP) has been described with invasive and non-invasive NAVA ${ }^{9-12}$. The breakpoint is the point at which the NAVA support is at a level where the patient's diaphragm is adequately unloaded. In order to locate this breakpoint for a specific patient, the NAVA level has to be increased progressively. With a given Edi, increasing NAVA levels will result in increasing peak inspiratory pressures (PIP). Once the breakpoint is reached, the respiratory muscle unloading is sufficient and further increase in NAVA level will not result in a higher PIP, because there will be a suppression of the patient's inspiratory drive. This NAVA level could therefore be considered as the level of "optimal unloading" of the diaphragm and hence, used as initial setting when starting NAVA. Firestone et al were the first to demonstrate that this neural feedback mechanism is intact in preterm infants ventilated with NAVA or NIV-NAVA ${ }^{9}$. A later study showed that the breakpoint changes before and after extubation from NAVA to NIV-NAVA ${ }^{11}$. In the study by Nam et al these findings were not confirmed in preterm patients on invasive NAVA. They concluded that neural feedback is insufficient in preterm infants during $\mathrm{NAVA}^{10}$. All these studies included preterm infants with various diseases and various postnatal ages. Data on preterm infants in the acute phase of RDS are lacking. These infants could eventually benefit a lot from optimal respiratory support in order to prevent intubation and other complications of RDS. Our current approach is to start with a NAVA level between 1 and $2 \mathrm{cmH}_{2} \mathrm{O} / \mu \mathrm{V}$ and then titrating the NAVA level up or down depending on the Edi peak levels, partial carbon dioxide pressure (pCO2) and signs of respiratory distress. Target Edi peak levels are between 10 and $15 \mu \mathrm{V}$. NAVA levels beyond $2 \mathrm{cmH}_{2} \mathrm{O} / \mu \mathrm{V}$ are rarely used.

The objective of this study was to investigate if a breakpoint could be determined in preterm infants in the early phase of acute RDS on invasive or non-invasive NAVA. This could help us to define the ideal and individual starting settings for NAVA in these infants.

\section{Materials and methods}

This was a prospective study conducted in the neonatal intensive care unit at the Universitair Ziekenhuis Brussel between December 2018 and June 2020. The hospital's medical ethics committee approved the study and parental consent was obtained prior to inclusion in the study. The study was registered on clinicaltrials.gov (NCT03780842).

The study population consisted of premature neonates born before 37 weeks of gestation supported with invasive or non-invasive NAVA for RDS. Patients were included within 24 hours after starting NAVA or NIV-NAVA if they were clinically stable. Exclusion criteria included congenital malformations or abnormalities of the diaphragm or other parts of the respiratory system, more than $20 \%$ of time spent in back-up ventilation and hemodynamic instability or rapid respiratory deterioration on NAVA. The decision for NAVA as mode for respiratory support was made by the attending physician. Indications were primary respiratory support, CPAP-failure or as post-extubation support. All patients were ventilated with a Servo-n ventilator (Getinge $\Omega$ ).

\section{Titration protocol}

Before starting the protocol, the ventilator limit for peak inspiratory pressure (PIP) was set to $35 \mathrm{cmH}_{2} \mathrm{O}$ (the maximum pressure that can be delivered to the patient is cut off at $30 \mathrm{cmH}_{2} \mathrm{O}$ ). Other ventilator settings were left unchanged and were as clinically indicated. Ventilator settings were according to our standard of care and as following: positive end-expiratory pressure (PEEP) between 4 and $7 \mathrm{mmHg}$, apnea time 2 to 5 seconds, back-up ventilation with a PIP of maximum $15 \mathrm{cmH}_{2} \mathrm{O}$ and a rate between 30 and 50 breaths per minute. Fraction of inspired oxygen $\left(\mathrm{FiO}_{2}\right)$ was titrated to obtain oxygen saturation $\left(\mathrm{SpO}_{2}\right)$ between $90 \%$ and $95 \%$. 
As a starting point, the NAVA level was reduced to $0,5 \mathrm{cmH}_{2} \mathrm{O} / \mu \mathrm{V}$ for 3 minutes. Next, the level was increased with $0,5 \mathrm{cmH}_{2} \mathrm{O} / \mu \mathrm{V}$ every 3 minutes until a maximum NAVA level of $4,0 \mathrm{cmH}_{2} \mathrm{O} / \mu \mathrm{V}$ was reached. All other settings except for $\mathrm{FiO}_{2}$ were kept constant throughout the study. A previous study performed in neonates using a similar titration protocol showed that a 3-minute period was long enough to see a response in PIP and $\mathrm{Edi}^{9}$.

Heart rate (HR) and oxygen saturation were recorded from the bedside monitor every 30 seconds. Data regarding the ventilation parameters were downloaded from the ventilator with SERVO tracker software (Getingeß) and exported in an Excel file. These data include PIP, Edi peak and min, respiratory rate (RR), $\mathrm{FiO}_{2}$ and NAVA level.

After the titration was completed, the level was returned to the initial NAVA level.

Patient data regarding gestational age, birth weight, surfactant administration, respiratory support and lung ultrasound score (LUS) were extracted from the medical file of the patient ${ }^{13}$. The Edi-change or delta Edi (Edi peak - Edi min) was used for all analyses. The average over each 3 minute interval was used for all variables.

\section{Statistical analysis}

PIP, delta Edi and NAVA level were plotted on a curve with PIP and Edi on the Y-axis and NAVA level on the X-axis. Visual inspection of the curve by two independent examiners was used to determine the inflection point for PIP and Edi, this was then called the breakpoint ${ }^{9-11}$. In analogy with previous studies on this topic, data from the NIV-NAVA patients where than combined by aligning each variable for the breakpoint and then averaging the variables for each NAVA step above and below the breakpoint ${ }^{9,11}$.

Linear mixed models were used to explain the evolution of the patients' data of RR, HR, and oxygen saturation over consecutive NAVA levels, allowing for different trends before and after the breakpoint.

\section{Results}

Sixteen patients were enrolled in the study, three of which could not be included in the analyses due to technical defects.

Ten premature infants were studied on NIV-NAVA and three infants on invasive NAVA. The NIV-NAVA patients had an average gestational age at birth of $31,4+3,2$ weeks (range $254 / 7$ to $352 / 7$ weeks), average birth weight of $1615+609 \mathrm{~g}$ (range 780 to $2570 \mathrm{~g}$ ) and average age at enrolment of 1,3 $+0,7$ days (range 1 to 3 days). All but two received surfactant. The LUS was available for 4 patients and was on average 10,8 + 1,9 (range 8 to 12), corresponding to more severe $\mathrm{RDS}^{13}$. Seven patients received caffeine. Baseline settings ranged for NAVA level from 1 to $2 \mathrm{cmH}_{2} \mathrm{O} / \mu \mathrm{V}$ and for PEEP from 5 to $7 \mathrm{cmH}_{2} \mathrm{O}$. Four infants were on NIV-NAVA as primary respiratory support (all less than 30 weeks gestational age), five because of CPAP failure (all 32 weeks gestational age or more), and one as post-extubation respiratory support. Two patients needed to be intubated after the study due to respiratory failure on NIV-NAVA.

Only three patients were studied on invasive NAVA ventilation. Mean gestational age at birth was $32,3+$ 4,8 weeks (range $266 / 7$ to 36 weeks), mean birth weight $1888+996 \mathrm{~g}$ (range 825 to $2800 \mathrm{~g}$ ) and mean age at enrolment $3,7+2,1$ days (range 2 to 6 days). All received surfactant and one received caffeine. LUS were not available for these patients. NAVA level at study entry was $1,2 \mathrm{cmH}_{2} \mathrm{O} / \mu \mathrm{V}$ in all patients and PEEP ranged from 6 to $7 \mathrm{cmH}_{2} \mathrm{O}$. All three infants were on NAVA after a period of high frequency ventilation or conventional volume-targeted ventilation. One patient had a pneumothorax for which a chest tube was inserted prior to study participation.

Figures 1 and 2 show changes in Edi and PIP according to the NAVA level for a representative patient on NIV-NAVA and invasive NAVA, respectively. As the NAVA level is increased, PIP increases accordingly. At a specific NAVA level, further increases are no longer associated with increases in PIP, and Edi values start do decrease. The point at which this happens is called the breakpoint and could be identified in all patients 
by visual inspection of the curves as previously validated ${ }^{9}$. In most cases there was agreement between the two interpreters. If not, this was easily solved by discussion.

Figure 3 shows the composite data of PIP and Edi for all patients on NIV-NAVA. The average breakpoint in the NIV-NAVA group was $2,35+0,63 \mathrm{cmH}_{2} \mathrm{O} / \mu \mathrm{V}$ (range 1,5 to $3 \mathrm{cmH}_{2} \mathrm{O} / \mu \mathrm{V}$ ). Because of the small number of patients in the invasive NAVA group $(n=3)$, we did not interpret the composite data but present the individual characteristics of these patients in table 1.

Respiratory rate, heart rate, oxygen saturation and $\mathrm{FiO}_{2}$ were analysed for all NIV-NAVA patients. The average respiratory rate was $67,3+4,6$ per minute, reflecting mild tachypnea compatible with a population of preterm infants with RDS. When plotting RR in time (figure 4), a significant decrease with increasing NAVA levels $(p<0,0001)$ was seen. On average, the RR decreased with 10 breaths per minute over the entire titration protocol, and this decrease was similar before and after the breakpoint. Further inspection of the individual curves highlighted a strong inter-patient variability of RR and in one study patient, the RR showed even a rising trend. HR was always within normal range (100 to $180 \mathrm{bpm}$ ) during the titration protocol, no bradycardic events occurred. There was no significant difference between the HR at the start and at the end of the titration protocol. There was a significant curvilinear trend, but this was not clinically relevant. Oxygen saturation was on average $95,6+1,1 \%$. In two patients, mild desaturation occurred with a minimal oxygen saturation of $86 \%$ for less than 30 seconds. For most patient $\mathrm{FiO}_{2}$ was stable during the protocol with an average $\mathrm{FiO}_{2}$ of $24,6 \%+2$, 0 . In one patient (GA 35 weeks, BW 2240g), with severe RDS, a temporary rise in $\mathrm{FiO}_{2}$ from $38 \%$ to $55 \%$ was needed to keep the oxygen saturation within the target range, this was seen at levels 2 and $2,5 \mathrm{cmH}_{2} \mathrm{O} / \mu \mathrm{V}$.

During the titration protocol no severe complications were seen for the NIV-NAVA patients. In the NAVAgroup one patient (GA 26 6/7w, BW 850g, postnatal day 2) did not support a NAVA level of $0,5 \mathrm{cmH}_{2} \mathrm{O} / \mu \mathrm{V}$. At this low level he exhibited tachycardia and tachypnoea followed by desaturation, therefore the patient only stayed on this level for 2 minutes instead of 3 . When he progressed to level $1 \mathrm{cmH}_{2} \mathrm{O} / \mu \mathrm{V}$ he recovered immediately and the titration protocol was completed as planned.

\section{Discussion}

This is the first study exploring the effect of increasing NAVA levels in premature neonates in the early phase of their RDS. Both with invasive as well as non-invasive NAVA, a breakpoint could be identified, which is in line with previous reports in premature neonates at an older age and with other conditions ${ }^{9-11}$. This study showed that also in the acute phase of RDS, premature neonates demonstrate a two-phased response to increasing NAVA levels. In the first phase there is an increase in PIP with increasing NAVA levels until the breakpoint is reached. In the second phase, when levels are further increased beyond this breakpoint, a stabilisation of the PIP is seen. This can be explained by the fact that using NAVA-levels beyond the breakpoint result in a decrease in Edi due to a suppression of spontaneous respiratory effort. This unloading of the respiratory muscles has previously been described in premature neonates beyond the acute phase of RDS and before and after extubation ${ }^{9,11}$. In our study, the decrease in Edi was rather subtle in the patients who were ventilated non-invasively. This means that the neural respiratory drive remains high even with NAVA levels beyond the breakpoint. We hypothesize that this is due to the relative inefficacy of non-invasive ventilation in premature neonates with large air leaks at the nasal interface. This was also seen in a previous titration study where neonates were studied before and after extubation ${ }^{11}$. With lower NAVA levels not all patients showed an important rise in Edi. Nevertheless, the low NAVA levels were well tolerated except in one patient. This could reflect an immaturity of the neural respiratory drive in our population of premature infants with acute RDS. Another possible explanation is that the duration of the interval between changes of NAVA-level (3 minutes) was too short to induce an adequate increase in respiratory drive. This hypothesis is supported by the fact that in the analysis of the respiratory rate we observed a within level rise in respiratory rate in the levels below the breakpoint in some patients. In the 3-minute interval respiratory rate continued to rise without reaching a plateau, suggesting that the interval was possibly too short to fully compensate for the suboptimal respiratory support (E-image 1). Similarly, in the study by Lee et al., where longer intervals of 10 minutes were used, a significant rise in Edi was detected in the lower NAVA levels ${ }^{10}$. 
The plateau PIP never exceeded $20 \mathrm{cmH}_{2} \mathrm{O}$ on non-invasive ventilation although the peak pressure limit was set at $35 \mathrm{cmH}_{2} \mathrm{O}$, allowing pressures up to $30 \mathrm{cmH}_{2} \mathrm{O}$. For the patients on invasive NAVA ventilation the maximum plateau PIP was $22 \mathrm{cmH}_{2} \mathrm{O}$. This suggests that in this population of premature neonates with acute RDS a protection mechanism for overdistension of the lung is functional. The physiological mechanism responsible for this protection is still unknown. The Hering-Breuer reflex could possibly explain this, although this is not confirmed in neonates. Also other feedback mechanisms might contribute to this complex feedback mechanism ${ }^{9}, 12,14$.

Most of the neonates were tachypnoeic during the trial, reflecting the population of neonates with acute RDS. In the NIV-NAVA patients, RR decreased significantly with on average 10 breaths per minute between the lowest and the highest NAVA levels. This reflects a decrease in work of breathing with higher NAVA levels. There were no significant changes in $\mathrm{HR}, \mathrm{SpO}_{2}$ and $\mathrm{FiO}_{2}$ detected during the trial. Two patients showed short and mild desaturation during the protocol with minimum $\mathrm{SpO}_{2}$ of $86 \%$ for 30 seconds. This was observed in the higher NAVA levels so probably due to the instable respiratory condition rather than the titration protocol itself. We could therefore conclude that the titration protocol and short periods of over- and undersupport were well tolerated.

The breakpoint in our study patients seemed to be at considerably higher levels than the levels that are commonly used to support similar patients in our unit. Prior to enrolment, patients on NIV-NAVA were on levels ranging from 1 to $2 \mathrm{cmH}_{2} \mathrm{O} / \mu \mathrm{V}$, while breakpoints were on average 1 level higher. Although this reflects suboptimal unloading of respiratory effort, only two patients subsequently needed to be intubated due to respiratory failure. Further research is needed to investigate whether the use of higher initial NAVA-levels aiming at optimal unloading is superior to the current approach.

A limitation of the study is the small number of patients on invasive ventilation. Enrolment of these patients was challenging, firstly, because parents often had to be approached for consent on the first postnatal day which was emotionally difficult, and secondly, because most premature infants with acute RDS are nowadays managed with non-invasive respiratory support combined with less invasive surfactant administration.

Due to the small sample size the statistical analyses for the NIV-NAVA patients are vulnerable to outliers. Although a significant decline in RR over the study period was detected, the rise in RR for one patient remains difficult to assign and does influence the analysis. Furthermore, because each patient was measured intensively, even the tiniest within patient change was detected, so we focused on the average change in RR over time. Another limitation of the analyses on RR, $\mathrm{HR}$ and $\mathrm{SpO}_{2}$ in relation to the changing NAVA levels is that they are confounded with time, as their order was the same for all patients. We did not look for the effect of a specific NAVA level or change in NAVA level on RR.

Information on partial carbon dioxide pressures or on tidal volumes during the titration procedure is lacking. The effect of changing NAVA levels on alveolar minute ventilation remains therefore unknown. Tidal volumes are difficult to measure reliably during non-invasive ventilation due to the large air leaks.

\section{Conclusion}

This study showed that preterm infants also in their early phase of RDS have a similar pattern of respiratory unloading as previously described in preterm infants at an older age and with other conditions. The levels at which maximal respiratory unloading was achieved during non-invasive NAVA were higher than the levels that are commonly used in this clinical situation.

Further studies are needed to evaluate the possible benefits and safety of the use of optimized NAVA levels in this population, both on short-term as well as on long-term clinical outcomes.

\section{Acknowledgments}

The authors declare that there is no conflict of interests.

\section{References}


1. Beck J, Emeriaud G, Liu Y, Sinderby C. Neurally adjusted ventilatory assist (NAVA) in children: a systematic review. Minerva Anestesiologica 2015;82(8):874-83.

2. Stein H, Howard D. Neurally adjusted ventilatory assist in neonates weighing $<1500$ grams: a retrospective analysis. J Pediatr 2012;160(5):786-9 e1.

3. Longhini F, Ferrero F, De Luca D, Cosi G, Alemani M, Colombo D, Cammarota G, Berni P, Conti G, Bona G, et al. Neurally adjusted ventilatory assist in preterm neonates with acute respiratory failure. Neonatology 2015;107(1):60-7.

4. Protain AP, Firestone KS, McNinch NL, Stein HM. Evaluating peak inspiratory pressures and tidal volume in premature neonates on NAVA ventilation. Eur J Pediatr 2020.

5. Lee J, Kim HS, Jung YH, Shin SH, Choi CW, Kim EK, Kim BI, Choi JH. Non-invasive neurally adjusted ventilatory assist in preterm infants: a randomised phase II crossover trial. Arch Dis Child Fetal Neonatal Ed 2015;100(6):F507-13.

6. Beck J, Reilly M, Grasselli G, Mirabella L, Slutsky AS, Dunn MS, Sinderby C. Patient-ventilator interaction during neurally adjusted ventilatory assist in low birth weight infants. Pediatr Res 2009;65(6):663-8.

7. Greenough A, Rossor TE, Sundaresan A, Murthy V, Milner AD. Synchronized mechanical ventilation for respiratory support in newborn infants. Cochrane Database Syst Rev 2016;9:CD000456.

8. Lemyre B, Laughon M, Bose C, Davis PG. Early nasal intermittent positive pressure ventilation (NIPPV) versus early nasal continuous positive airway pressure (NCPAP) for preterm infants. Cochrane Database Syst Rev 2016;12:CD005384.

9. Firestone KS, Fisher S, Reddy S, White DB, Stein HM. Effect of changing NAVA levels on peak inspiratory pressures and electrical activity of the diaphragm in premature neonates. J Perinatol 2015;35(8):612-6.

10. Nam SK, Lee J, Jun YH. Neural feedback is insufficient in preterm infants during neurally adjusted ventilatory assist. Pediatr Pulmonol 2019;54(8):1277-83.

11. LoVerde B, Firestone KS, Stein HM. Comparing changing neurally adjusted ventilatory assist (NAVA) levels in intubated and recently extubated neonates. J Perinatol 2016;36(12):1097-100.

12. Lecomte F, Brander L, Jalde F, Beck J, Qui H, Elie C, Slutsky AS, Brunet F, Sinderby C. Physiological response to increasing levels of neurally adjusted ventilatory assist (NAVA). Respir Physiol Neurobiol $2009 ; 166(2): 117-24$.

13. Brat R, Yousef N, Klifa R, Reynaud S, Shankar Aguilera S, De Luca D. Lung Ultrasonography Score to Evaluate Oxygenation and Surfactant Need in Neonates Treated With Continuous Positive Airway Pressure. JAMA Pediatr 2015;169(8):e151797.

14. Leither J, Manning H. The Hering-Breuer reflex, feedback control, and mechanical ventilation: the promise of neurally adjusted ventilatory assist. Crit Care Med 2010;38:1905-6. 

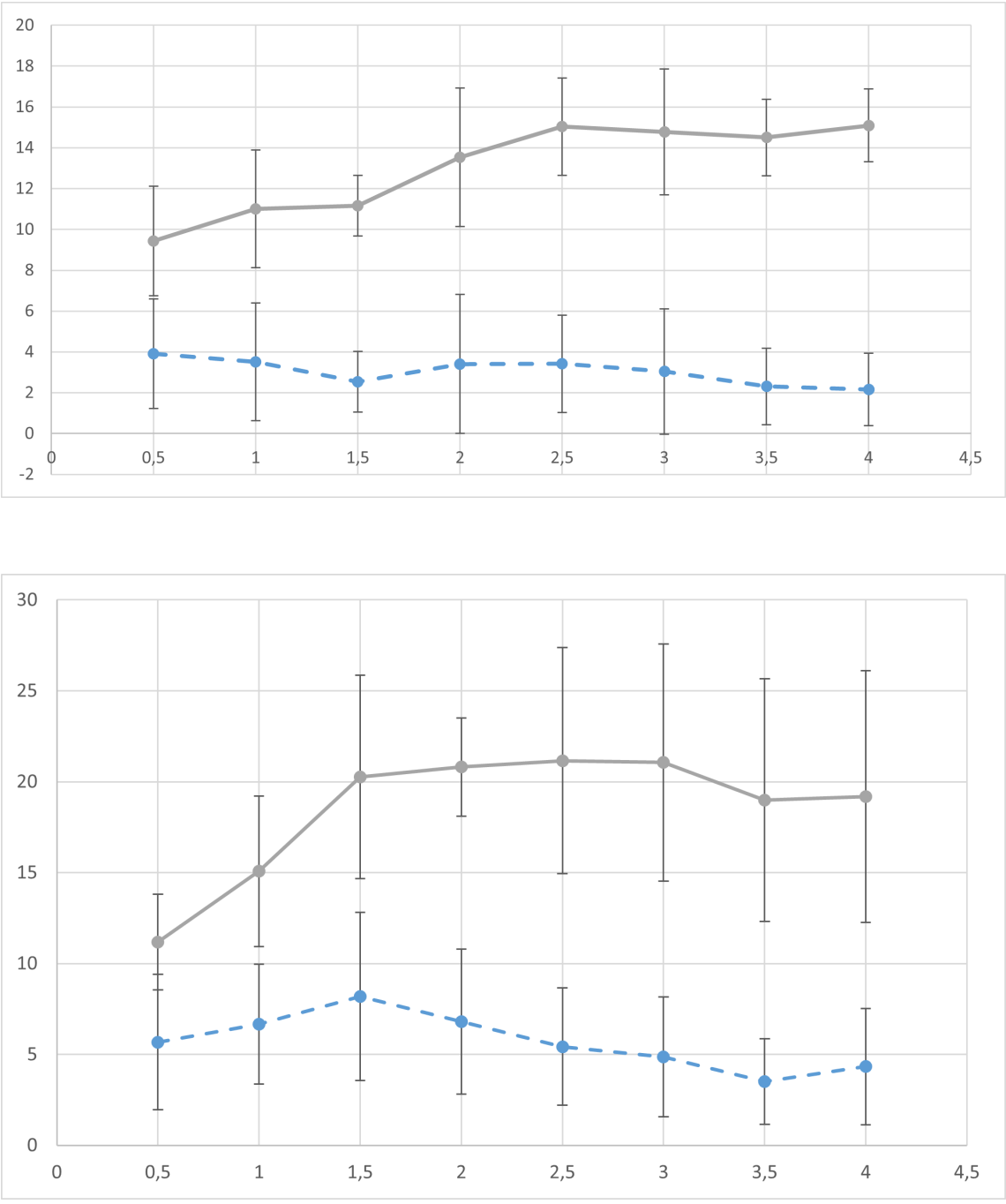

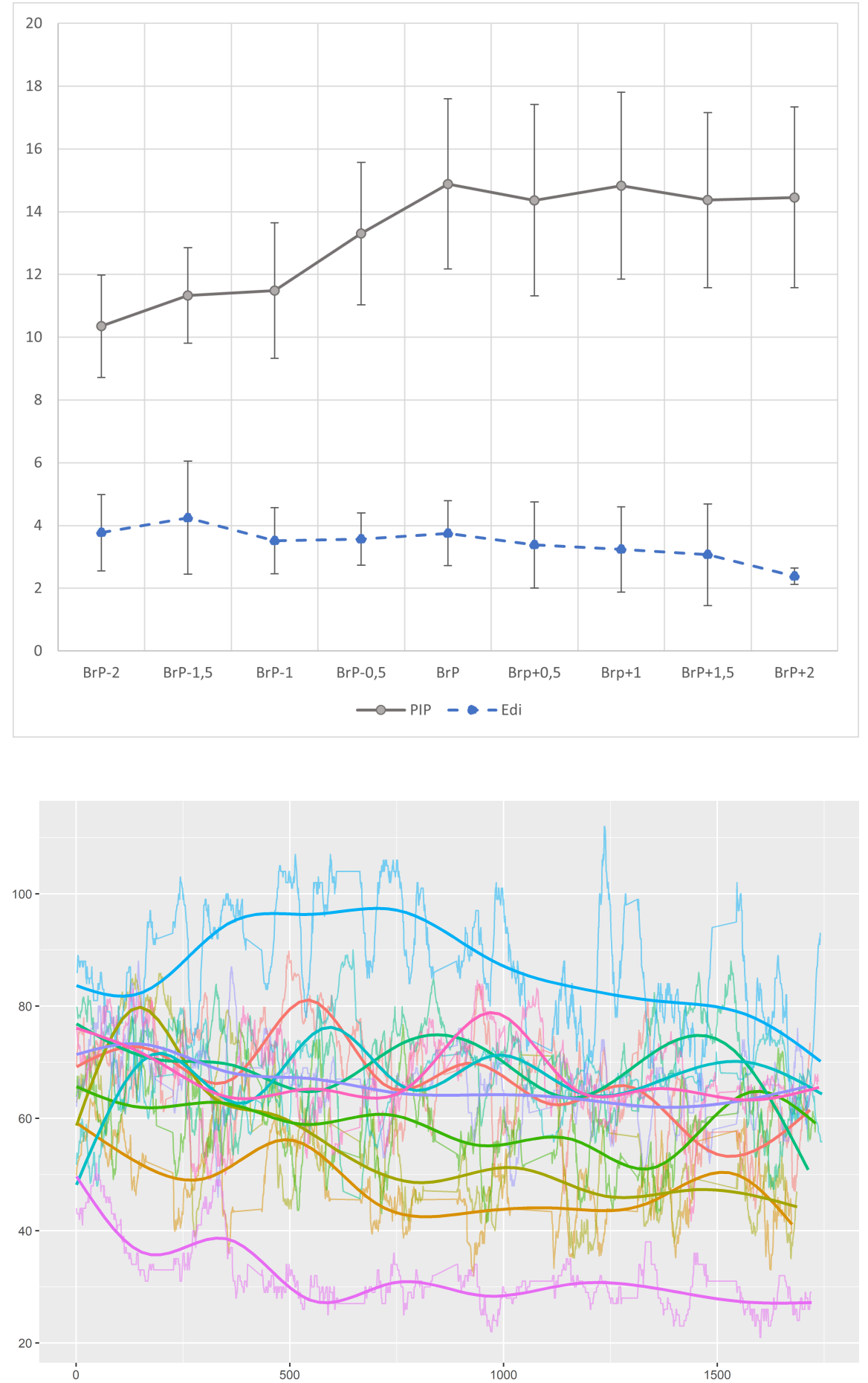

\section{Hosted file}

Table.pdf available at https://authorea.com/users/383486/articles/499356-effect-of-changingnava-levels-in-preterm-infants-with-rds 